\title{
Macrophage activation syndrome: a potentially fatal complication of rheumatic disorders
}

\author{
S Sawhney, P Woo, K J Murray
}

\begin{abstract}
Aims-To review the precipitating events, clinical features, treatment, and outcome of macrophage activation syndrome (MAS).

Methods-Retrospective review of cases of MAS from a prospectively collected database of children with rheumatic diseases from 1980 to 2000 .

Results-Nine patients (eight girls) were considered to have evidence of MAS. The primary diagnosis was systemic onset juvenile idiopathic arthritis in seven, enthesitis related arthritis in one, and chronic infantile neurological cutaneous articular syndrome in one. Mean age of onset was 5.7 years, and duration prior to MAS, 4.2 years. No medication was identified as a trigger. Eight had infections prior to MAS; specific infectious agents were identified in four. High grade fever, new onset hepatosplenomegaly, and lymphadenopathy were common clinical features. Platelet counts fell dramatically, from an average of 346 to $99 \times 10^{9} / 1$. Mean erythrocyte sedimentation rate (in three patients) fell from 115 to $28 \mathrm{~mm} / \mathrm{h}$. Eight had abnormal liver function during the disease course, and six had coagulopathy. Bone marrow examination supported the diagnosis with definite haemophagocytosis in four of seven. All received high dose steroids (eight intravenous, one oral), five cyclosporin, two cyclophosphamide, and one antithymocyte globulin. Two of three patients with significant renal impairment died.

Conclusion-MAS is a rare and potentially fatal complication of childhood rheumatic disorders. Most of our patients were female, and most cases were preceded by infection. Bone marrow studies support the diagnosis. Deranged renal function may be a poor prognostic sign. Aggressive early therapy is essential. (Arch Dis Child 2001;85:421-426)
\end{abstract}

Keywords: macrophage activation syndrome; infection; arthritis; rheumatic disease

Department of Rheumatology, Great Ormond Street Hospital, London WC1N 3JH, UK

S Sawhney

$\mathrm{P}$ Woo

K J Murray

Correspondence to: Dr Murray

k.murray@ich.ucl.uk

Accepted 13 June 2001

Although macrophage disorders are usually a consequence of a decrease in function, an increase in macrophage activity can also have adverse consequences for individuals. Macrophage activation syndrome (MAS) is a clinical syndrome caused by excessive activation and proliferation of well differentiated macrophages. This condition occurs in a heterogeneous group of diseases, ranging from infections or neoplasm to haematological conditions and rheumatic disorders. ${ }^{1}$ A series of patients with benign histiocytic proliferation, distinct from malignant histiocytosis was first described by Risdal et al in 1979, who postulated that this condition occurred secondary to an immune dysregulation induced in response to viral infections in immunocompromised hosts. ${ }^{2}$ The term MAS was coined by Hadchouel et al in 1985 in their description of seven patients with systemic onset juvenile idiopathic arthritis (SOJIA) who developed this complication during the course of their disease. ${ }^{3}$ The clinical findings of MAS are dramatic. Typically patients with a chronic disease become acutely ill at presentation with persistent fever, lymphadenopathy, and hepatosplenomegaly. Profound depression of one or more blood cell lines, low erythrocyte sedimentation rate (ESR), raised liver cell enzymes, and abnormalities of the clotting profile commonly occur. ${ }^{1}$ The pathognomonic feature of this syndrome is seen on bone marrow examination: numerous well differentiated macrophages actively phagocytosing haematopoetic elements. ${ }^{4}$ Such cells may be found in various organs and may be responsible for many of the systemic features in this condition. Goldberg and Nezelof reviewed 38 cases of lymphohistiocytosis (considered equivalent to MAS); they found that macrophages are activated, with expression of OKM1, OKT9, and HLA DR, and show either patent or occult erythrophagocytosis. ${ }^{5}$ MAS in the context of rheumatological conditions occurs most commonly in SOJIA, but may occur in other forms of juvenile idiopathic arthritis (JIA), or indeed in other systemic rheumatic disorders. ${ }^{6}$ The specific aetiology of this condition as a secondary phenomenon is not well understood.

To date only case reports of relatively small numbers of patients, and some pooled patients have been reported. ${ }^{36-9}$ This report presents a retrospective review of all patients in our unit (a tertiary level paediatric rheumatology unit) over the past 20 years. To our knowledge this is the largest reported series of MAS in paediatric rheumatological disorders occurring in a single unit. One of the patients has been reported previously. ${ }^{10}$

\section{Methods}

All cases considered to have clinical and laboratory evidence of MAS were retrospectively reviewed. A total of nine patients were identified from a prospectively collected database of paediatric rheumatology patients from 1980 to 2000 . Systematic review of the case notes of all patients was undertaken. All 
Table 1 Demographics, primary disease, and pre-MAS clinical features

\begin{tabular}{|c|c|c|c|c|c|c|}
\hline Patient & Diagnosis & Sex & $\begin{array}{l}\text { Age (y) at onset } \\
\text { of primary } \\
\text { disease }\end{array}$ & $\begin{array}{l}\text { Disease } \\
\text { duration } \\
\text { pre-MAS (y) }\end{array}$ & $\begin{array}{l}\text { Pre-MAS } \\
\text { polyarthritis }\end{array}$ & Drugs pre-MAS \\
\hline 1 & SOJIA & $\mathrm{F}$ & 9.8 & 0.8 & + & Antibiotic \\
\hline 2 & ERA & M & 11.7 & 5.4 & + & Prednisolone/indomethacin \\
\hline 3 & SOJIA & $\mathrm{F}$ & 4.0 & 13.9 & + & Methotrexate \\
\hline 4 & SOJIA & $\mathrm{F}$ & 1.7 & 0.1 & + & Antibiotic \\
\hline 5 & SOJIA & $\mathrm{F}$ & 13.0 & 9.0 & + & Prednisolone \\
\hline 6 & SOJIA & $\mathrm{F}$ & 6.5 & 1.5 & + & Prednisolone/naproxen \\
\hline 7 & CINCA & $\mathrm{F}$ & 0.6 & 1.9 & + & Antibiotic/naproxen \\
\hline 8 & SOJIA & $\mathrm{F}$ & 1.9 & 1.0 & + & Naproxen \\
\hline 9 & SOJIA & $\mathrm{F}$ & 2.0 & 0.2 & + & Antibiotic/indomethacin \\
\hline
\end{tabular}

SOJIA, systemic onset juvenile idiopathic arthritis; ERA, enthesitis related arthritis; CINCA, chronic infantile neurological cutaneous articular syndrome.

relevant clinical and laboratory features, treatment, and outcome were recorded. Laboratory data were collected at the time of onset of MAS (clinically determined), and thereafter as clinically needed. Comparisons were made on a weekly basis, where available until clinical and laboratory resolution of MAS. As the total number of patients was nine, only descriptive statistical analysis was applied.

\section{Results}

DEMOGRAPHICS AND DISEASE TYPE

Over the past 20 years the unit has had 103 new patients with SOJIA, 37 with enthesitis related arthritis (ERA), and three with the chronic infantile neurological cutaneous articular (CINCA) syndrome (see table 1). Nine patients developed MAS during the course of their primary rheumatic disease, of which eight were female. Seven patients had SOJIA, one ERA, and one the CINCA syndrome. The duration of primary disease prior to development of MAS averaged 4.2 years; MAS occurred either during the initial presentation, or up to nearly 14 years after diagnosis. Mean age at primary disease onset (of the rheumatic disorder) was 5.7 years. In the weeks prior to MAS all patients were noted to have active polyarticular disease, with laboratory testing showing significantly raised inflammatory markers.

\section{TRIGGERS AND CLINICAL FEATURES OF MAS}

All patients had active systemic rheumatic disease prior to developing MAS. Patients 4 and 9 had MAS during the first presentation of their primary condition. Eight patients had a possible or definite infection four to eight weeks prior to developing MAS, and of these four had specific infections (see table 2). Patient 7 had the CINCA syndrome. She was dependent on total parenteral nutrition and developed a central line infection with blood cultures positive for enterococcus, at the time of developing MAS. Four of the eight patients had been treated with antibiotics for presumed sepsis, of which only patient 7 had positive blood cultures. No specific drugs were identified as an MAS trigger. In addition there had been no change in the drugs that the patients had been taking in the week before development of MAS. No patient had been on gold, sulphasalazine, or penicillamine (previously reported as triggers of MAS). Patient 3 was on methotrexate, and had stopped cyclosporin two weeks prior to developing MAS. Similarly patient 2 had stopped methotrexate four months prior to developing MAS. High grade fever and organomegaly were the hallmarks of clinical examination. Lymphadenopathy was seen in six and icterus in two. Three patients had a stormy clinical course with the need for intensive care support, including ventilation. Four patients with SOJIA had an erythematous rash and only one patient had frank petechiae. Two patients had clinical evidence of CNS involvement with irritability and altered mental status.

\section{LABORATORY DATA}

Pre-MAS

In the four to eight weeks preceding the onset of MAS, detailed laboratory results were available for eight patients (see table 3). Mean ESR was $106.5 \mathrm{~mm} / \mathrm{h}$ (range 83-139), and mean platelet count $346 \times 10^{9} / 1$ (range 134-510). Abnormal biochemistry was not a common feature in the weeks preceding MAS. Only patients 4 and 8 had moderate elevation of liver cell enzymes.

\section{MAS onset}

Paired data on the change in haemoglobin, platelets, and white cell count were available in eight patients. No significant change occurred

Table 2 Triggers and clinical features of MAS

\begin{tabular}{|c|c|c|c|c|c|c|}
\hline \multirow[b]{2}{*}{ Patient } & \multirow{2}{*}{$\begin{array}{l}\text { Pre-MAS } \\
\text { Trigger }\end{array}$} & \multicolumn{5}{|c|}{ Clinical features of $M A S$} \\
\hline & & Persistent fever & Hepatosplenomegaly & Lymphadenopathy & Icterus & Ventilation/PICU \\
\hline 1 & Diarrhoea & + & + & + & + & + \\
\hline 2 & EBV & + & + & + & - & - \\
\hline 3 & - & + & + & - & - & - \\
\hline 4 & Diarrhoea & + & + & + & - & - \\
\hline 5 & EBV & + & + & - & - & - \\
\hline 6 & URTI & + & - & - & - & - \\
\hline 7 & Enterococcal sepsis & + & + & + & - & + \\
\hline 8 & Diarrhoea & + & + & + & - & - \\
\hline 9 & Varicella & + & + & + & + & + \\
\hline
\end{tabular}

PICU, paediatric intensive care unit; EBV, Epstein-Barr virus; URTI, upper respiratory tract infection. 
Table 3 Comparative laboratory data pre-MAS and during $M A S$

\begin{tabular}{|c|c|c|c|c|}
\hline Data & Pre-MAS & MAS onset & $M A S$ course (wk 1) & $\begin{array}{l}\text { Total no. of patients with } \\
\text { abnormalities during the } \\
M A S \text { episode }\end{array}$ \\
\hline $\mathrm{Hb}, \mathrm{g} / \mathrm{l}$ (mean) & 8.9 & 9.2 & 8.9 & 8 \\
\hline WBC $\times 10^{9}$ (mean) & 12.5 & 10.1 & 7.8 & 8 \\
\hline Platelets $\times 10^{9}$ (mean) & 346 & 99 & 105 & 8 \\
\hline $\mathrm{ESR}, \mathrm{mm} / \mathrm{h}$ (mean) & $115 t$ & $28+$ & - & 3 \\
\hline Liver function tests ${ }^{\star}$ & $2 \ddagger$ & 6 & 2 & 8 \\
\hline Coagulopathy ${ }^{\star}$ & 0 & 5 & 1 & 6 \\
\hline Reduced fibrinogen & 0 & 1 & 1 & 2 \\
\hline Renal function ${ }^{\star}$ & 0 & 1 & 2 & 3 \\
\hline Bone marrow (number of patients) & 0 & \multicolumn{2}{|c|}{$\begin{array}{l}7 \text { patients had a bone marrow aspirate } \\
\text { during the MAS episode }\end{array}$} & $\begin{array}{l}5 \text { (including } 1 \text { postmortem } \\
\text { specimen) }\end{array}$ \\
\hline
\end{tabular}

^Number of patients with abnormal results.

†Paired data on three patients.

$\ddagger$ Mild elevation of liver enzymes.

in haemoglobin, from an average of $8.9 \mathrm{~g} / 1$ prior to developing MAS, to a mean of $9.2 \mathrm{~g} / 1$ at the onset of MAS. White blood cell (WBC) count fell from 12.5 to $10.1 \times 10^{9} / 1$. However, the platelet count in these eight patients fell from a mean of 346 to $99 \times 10^{9} / 1$. Paired ESR data were available in only three (patients 3,7 , and 8 ) and dropped from a mean of 115 to $28 \mathrm{~mm}$ in the first hour. Eight patients had coagulation studies at onset, of which five were abnormal. Fibrinogen had been assayed in six patients at disease onset and was decreased in one. Six patients had abnormal liver function tests at disease onset: bilirubin was raised in three patients, aspartate transaminase (AST) in three, and alanine transaminase (ALT) in five. Urea and creatinine were raised in only one patient at the onset of MAS. Bone marrow study was carried out in seven patients; haemophagocytosis was confirmed in four. Interestingly patient 9 had a normal bone marrow study, but autopsy examination a few days later revealed haemophagocytosis in the liver, spleen, lymph node, and bone marrow.

\section{MAS course}

On day 7 of MAS, mean haemoglobin was 8.9 $\mathrm{g} / 1$; mean WBC and platelet counts were $7.8 \times$ $10^{9} / 1$ and $105 \times 10^{9} / 1$ respectively. Patients 2 and 5 developed evidence of hepatitis, taking the total with hepatic derangement to eight. Patient 9 underwent clotting studies three days after onset; these were abnormal, taking the total number with coagulopathy to six. One patient developed a reduction in fibrinogen concentration, interestingly without any change in clotting studies. Two patients developed a derangement in renal function, taking the total number to three. Two of three patients with renal impairment subsequently died.

Table 4 MAS outcome data

\begin{tabular}{llllll}
\hline Patient & $\begin{array}{l}\text { Clinical recovery } \\
\text { (days) }\end{array}$ & $\begin{array}{l}\text { Haematological } \\
\text { recovery (days) }\end{array}$ & $\begin{array}{l}\text { Biochemical } \\
\text { recovery (days) }\end{array}$ & $\begin{array}{l}\text { Delay to } \\
\text { diagnosis (days) }\end{array}$ & Outcome \\
\hline 1 & - & - & - & 04 & Died \\
2 & 35 & 35 & 77 & 21 & Recovered \\
3 & 4 & 11 & 28 & 02 & Recovered \\
4 & Unclear & 11 & 21 & $\star$ & Recovered \\
5 & 48 & NA & NA & 06 & Recovered \\
6 & 14 & 14 & 14 & 04 & Recovered \\
7 & 27 & 19 & 16 & 01 & Recovered \\
8 & 14 & 14 & 23 & 01 & Recovered \\
9 & - & - & - & 07 & Died \\
\hline
\end{tabular}

^Diagnosis made retrospectively, based on review of bone marrow aspirate 4 months later. NA, not available.

\section{MAS treatment}

All patients were treated with steroids: eight received intravenous methylprednisolone, and one oral prednisolone. Methylprednisolone was given at a dose of $30 \mathrm{mg} / \mathrm{kg} /$ day (maximum $1 \mathrm{~g}$ ); patients received 3-10 doses during the course of their disease. Five patients received cyclosporin (dose range $2-5 \mathrm{mg} / \mathrm{kg} /$ day) and one was treated with a combination of etoposide, cyclosporin, and intravenous methylprednisolone. Two patients were treated with cyclophosphamide: patient 4 , who was initially thought to have polyarteritis nodosa, and patient 1 who had severe renal impairment and in whom cyclosporin was considered contraindicated.

\section{MAS OUTCOME}

Table 4 presents details of MAS outcome. Two patients died in hospital, both of whom had been treated for multiorgan failure in intensive care. The overall mortality was thus $22 \%$. Mean time to clinical recovery was clear in six of seven survivors and averaged 23.6 days; haematological recovery occurred in an average of 17.3 days and biochemical recovery in an average of 30 days. Patient 6 recovered clinically in 14 days, but on day 32 after the first episode developed streptococcal sepsis and a second episode of MAS.

\section{Discussion}

JIA is the umbrella term under which most childhood chronic arthritides of unknown cause are considered. ${ }^{11}$ This term has now replaced juvenile chronic arthritis/juvenile rheumatoid arthritis in the new classification system proposed by the International League of Associations of Rheumatologists. ${ }^{12}$ SOJIA represents about $10-20 \%$ of all forms of JIA. The clinical features of SOJIA itself may be driven by proinflammatory cytokines. ${ }^{13} \mathrm{Al}-$ though MAS can occur in any systemic rheumatological disorder, it is most common in this condition.

The term reactive haemophagocytic lymphohistiocytosis (RHLH) is also used to describe this condition. ${ }^{1}$ Haemophagocytic lymphohistiocytosis (HLH) is a term which includes most of the patients with macrophage related disorders (class II histiocytosis) and contains two different conditions which may be 
Table 5 SOfIA versus MAS: clinical differences

\begin{tabular}{lll}
\hline & SOfIA flare & MAS \\
\hline Fever & Quotidian & Persistent \\
Lymphadenopathy & + & + \\
Hepatosplenomegaly & + & + \\
Others & Arthritis & Altered mental status \\
& Serositis & Petechial rash \\
WBC & Evanescent rash & \\
Haemoglobin & $\uparrow \uparrow$ & $\downarrow$ \\
Platelets & $\mathrm{N}$ & $\downarrow$ \\
ESR & $\uparrow \uparrow$ & $\downarrow$ \\
Bilirubin & $\uparrow \uparrow$ & $\downarrow$ \\
ALT/AST & $\mathrm{N}$ & $\uparrow$ \\
PT & $+/-$ & $\uparrow$ \\
PTTK & $\mathrm{N}$ & $\uparrow$ \\
Fibrinogen & $\mathrm{N}$ & $\uparrow$ \\
\hline
\end{tabular}

N, normal; PT, prothrombin time; PTTK, partial thromboplastin time with kaolin added.

difficult to distinguish from each other: primary or familial haemophagocytic lymphohistiocytosis (FHL); and secondary HLH, which includes infection/virus associated HLH (VAHLH) and malignancy associated haemophagocytic syndrome. ${ }^{14}$ Nomenclature is quite confusing, with RHLH and MAS being used interchangeably. MAS is similar to class II histiocytosis, or to "macrophage related disorders" as recently modified. ${ }^{14-16}$

Although MAS in systemic rheumatic diseases bears clinical and biological similarities to other haemophagocytic syndromes as detailed above, it is unique in three distinct ways:

(1) MAS appears to occur with varying degrees of severity, ranging from the moribund child with persistent high grade fever, significant hepatosplenomegaly, icterus, and laboratory parameters showing pancytopenia, coagulopathy, hepatic, and renal derangement to the unwell child with persistent fever, no significant organomegaly, a relative drop in blood cell lines, and mild if any coagulopathy. The biology behind these varied changes is poorly understood.

(2) MAS occurs in systemic rheumatic diseases, primarily SOJIA, which is an inflammatory disorder with haematological abnormalities reflecting this. Thus anaemia, leucocytosis, and thrombocytosis with a raised ESR and $C$ reactive protein are typical of active disease. A significant drop in these laboratory parameters, even though technically not in the range of thrombocytopenia or leucopenia, cannot be dismissed as irrelevant. It may well represent the onset of MAS in the correct clinical setting, and was seen in our patients as well. A "relative "change in counts may in fact be a key to early diagnosis and appropriate intervention.

(3) Early and aggressive immunosuppression is likely to benefit these patients, unlike in FHL where bone marrow transplantation is the cure.

It is therefore likely that no specified diagnostic criteria for MAS have been developed. A high index of suspicion in the clinical setting detailed earlier, with laboratory/bone marrow supportive features and appropriate therapy by an experienced team, are the key factors which determine outcome of this potentially fatal complication.

SOJIA is a condition characterised by repetitive disease flares. It is thus critical to differentiate a flare of the disease from the onset of MAS in this setting, as they have very different treatments and prognosis. Table 5 presents the key differences. ${ }^{1}$ Other important differential diagnoses would include sepsis, and side effects of medications. Thrombotic thrombocytopenic purpura is another important differential diagnosis, especially in the severe end of the spectrum of MAS; however, microangiopathic anaemia is a key feature in the former. ${ }^{17}$

This cohort was predominantly female, despite SOJIA having an equal sex distribution. Three of four cases in another series were females. ${ }^{18}$ The reason for this female preponderance is not understood; however, this was a small cohort of patients and further a pooled report of seven patients from France had a male preponderance. ${ }^{3}$

Clinical features and laboratory data in MAS are similar to those seen in VAHLH and FHL. ${ }^{214}$ VAHLH has been described in both patients without any preexisting disease and in immunosuppressed individuals. ${ }^{2}$ In this study 15 of the 19 patients had a confirmed viral infection (herpes group in 14 and adenovirus in one). Evidence suggests that virtually any infectious agent can precipitate MAS. ${ }^{4}$ Possible infections prior to MAS were seen in eight of the nine cases in our series; two had documented Epstein-Barr virus (EBV) infection. In previous case reports EBV infection has been cited as a common predisposing infective agent. $^{710}$ Of the nine patients we have described, one was on methotrexate and three on oral steroids. The remainder had been on nonsteroidal anti-inflammatory drugs (NSAIDs) only, yet developed MAS. It is noteworthy, however, that all our patients suffered from an underlying "immune mediated" disease.

The aetiology of this condition is not well understood and scientific investigation to date is limited. Prior to recognition of this condition, similar clinical features in patients with SOJIA were thought to be related to aspirin or Reye's syndrome. ${ }^{19}{ }^{20}$ In the 1980 s gold (sodium aurothiomalate) was used as a second line agent for JIA. Reports appeared of "consumptive coagulopathy", resulting in some fatalities when these children received the second dose of gold. ${ }^{21}$ The clinical features were in fact similar to MAS and four of the five patients in this report had SOJIA. Similarly, NSAID use or changes have been implicated previously, but the syndrome has been described in patients who were not on any NSAIDs. ${ }^{32}$

The clinical features and laboratory findings of FHL (a disease both clinically and haematologically similar to MAS) are compatible with the biological effects of several proinflammatory cytokines. Interferon gamma (IFN $\gamma$ ), tumour necrosis factor (TNF), and interleukin 6 have been shown to be increased in nine children with FHL. Soluble CD8 was also raised in this group of children, suggesting a role of cytotoxic $\mathrm{T}$ lymphocytes in the pathology of 
this condition. ${ }^{23}$ This author further speculated that IFN $\gamma$ with its macrophage activating capacity, stimulates the mononuclear phagocyte system and thus the release of monokines such as TNF, which in turn serve as important disease promoters. Studies from adult patients with $\mathrm{HLH}$ of various aetiologies also suggest that IFN $\gamma$ and macrophage colony stimulating factor, at least partly from $\mathrm{T}$ cells, might contribute to the activation of monocytes, resulting in upregulated monokine production and haemophagocytosis. ${ }^{24}$ Raised TNF $\alpha$ has been documented in few patients with MAS in the context of rheumatological conditions. ${ }^{18}$ Our study was a clinical review of the patients, and no specific biological markers were looked at. These findings are, however, very important and are likely to improve therapy of MAS in the coming years with, for instance, use of specific biological agents.

Rapidly evolving (pan)cytopenias should alert the clinician to this potential complication. ${ }^{4}$ In our series only platelet count had a dramatic fall, though the haemoglobin was already depressed in many patients. The aetiology for profound depletion of haemoglobin, WBCs, and platelets in these patients is thought to be multifactorial: haematophagocytosis, depression of progenitor cell proliferation, and the presence of inhibitory lymphokines are some of the key factors involved. ${ }^{242526}$

New onset coagulopathy is a characteristic feature of MAS, and seen in the majority of our patients, compatible with other reports..$^{20}$ Depression of fibrinogen concentrations has been said to be characteristic of MAS in one report, but was rare in our series. ${ }^{18}$ This difference may be explained by small patient groups, or under estimated as these patients usually have high fibrinogen concentrations. ${ }^{6}$ It has been suggested that many, if not all SOJIA patients have evidence of low grade vasculitis or incipient disseminated intravascular coagulation which may precede onset of $\mathrm{MAS}^{27}$ Actual coagulopathy in patients with SOJIA is, however, rare as shown both in our patients and in previous reports. ${ }^{28}$

Other potential biological markers of MAS, probably driven by cytokines, ${ }^{23}$ are triglycerides and ferritin, which were not studied in our patients.

In our series not all patients had demonstrable haemophagocytosis on bone marrow examination or on postmortem studies. No patient had hypocellular or dysplastic marrow. The frequency of documentation of this pathological phenomenon in MAS is variable, ${ }^{3618}$ and we believe it is dependent on the timing of the aspiration, and also on the spectrum of patients. It is critical to differentiate this condition from malignant disorders, which may be challenging, ${ }^{8}$ especially with the first presentation of the patient when arthritis is not obvious. Associated malignant diseases can be acute monocytic leukemia, chronic myelomonocytic leukemia, and malignant histiocytosis (MH). Though the nomenclature is confusing, $\mathrm{MH}$ is usually placed in the large but heterogeneous group of anaplastic large cell lymphomas. ${ }^{29}$

\section{Key messages}

- MAS is a rare complication of childhood rheumatic disease

- It is a potentially fulminant disorder, which may occur as part of the initial presentation of the rheumatic disease

- An infective trigger may herald the onset of this complication in predisposed patients

- Differentiation from a disease flare may be difficult, but is critical to ensure optimal outcome

- An early and dramatic fall in platelet count is characteristic, with changes in WBC and haemoglobin being more variable early and common

- Elevation in transaminases and coagulation abnormalities may not be present at onset of MAS

- Bone marrow examination is supportive, but false negative reports occur as a result of sampling errors or the subtle nature of the disease

- Multisystem involvement is a poor prognostic sign

High dose corticosteroid treatment is the suggested initial treatment of choice in MAS, ${ }^{3}$ but some patients seem to be corticosteroid resistant. ${ }^{16}$ Low dose steroids may not be effective, as has been suggested earlier. ${ }^{9}$ Successful treatment of Langerhan cell histiocytosis and MAS with cyclosporin suggests that $T$ lymphocytes are pivotal in the pathogenesis of this condition, as the drug is known to selectively suppress the function of $\mathrm{T}$ helper cells by suppressing proinflammatory cytokines. ${ }^{6}{ }^{18}{ }^{30}$ Recent published data have shown a raised TNF $\alpha$ in both SOJIA and in patients with MAS in systemic rheumatic diseases. ${ }^{31}$ This would suggest that anti-TNF therapy may be of benefit in treating patients with MAS, as has been shown in isolated case reports. ${ }^{32}$ Mortality in this condition is high. ${ }^{3}$ All five patients reported by Mouy et al survived, ${ }^{6}$ and none had renal involvement. In our cohort, two of the three children with renal impairment died, even though they were diagnosed early on in the course of this complication. Although the numbers are small, multisystem involvement certainly seems to be a poor prognostic feature in this condition. A review of 23 patients with histiocytosis also noted that that the four patients who died had rapidly progressive multisystem failure and succumbed to their illness within four weeks. ${ }^{4}$

CONCLUSION

MAS is a serious complication of systemic rheumatic disorders, primarily SOJIA, associated with morbidity and death. Early recognition and aggressive therapy are critical. Clinical differentiation from a typical flare of SOJIA is vital, as delay in specific therapy may prove deleterious. Further immunological and genetic studies are needed in larger cohorts of patients in order to better understand the 
pathophysiology of MAS. Only with this will come application of more appropriate and effective therapy. ${ }^{33}$ The recent discovery of perforin (a molecule which mediates the destruction of virus infected or transformed cells) gene mutations in both familial forms of haemophagocytosis and in patients with systemic onset JIA may have implications for the aetiology of this complication and indeed help with institution of new therapies. ${ }^{34} 35$

1 Grom AA, Passo M. Macrophage activation syndrome in systemic juvenile rheumatoid arthritis. $f$ Pediat 1996;129:750-4 [comment in f Pediatr 1997;130:1012].

2 Risdall RJ, McKenna RW, Nesbit ME, et al. Virusassociated hemophagocytic syndrome: a benign histiocytic proliferation distinct from malignant histiocytosis. Cancer 1979;44:993-1002.

3 Hadchouel M, Prieur AM, Griscelli C. Acute hemorrhagic, hepatic, and neurologic manifestations in juvenile rheumatoid arthritis: possible relationship to drugs or infection. $\mathcal{F}$ Pediatr 1985;106:561-6.

4 Reiner AP, Spivak JL. Hematophagic histiocytosis. A report of 23 new patients and a review of the literature. Medicine $1988 ; 67: 369-88$

5 Goldberg J, Nezelof C. Lymphohistiocytosis: a multifactorial syndrome of macrophagic activation: clinicopathological study of 38 cases. Hematol Oncol 1986;4:27589.

6 Mouy R, Stephan JL, Pillet P, et al. Efficacy of cyclosporine $A$ in the treatment of macrophage activation syndrome in juvenile arthritis: report of five cases. 7 Pediatr 1996;129. $750-4$

7 Davies SV, Dean JD, Wardrop CA, Jones JH. Epstein-Barr virus-associated haemophagocytic syndrome in a patient with juvenile chronic arthritis. $B r \not \mathcal{F}$ Rheumatol 1994:33. 495-7.

8 Heaton DC, Moller PW. Still's disease associated with Coxsackie infection and haemophagocytic syndrome. Ann Rheum Dis 1985;44:341-4.

9 Ravelli A, De Benedetti F, Viola S, Martini A. Macrophage activation syndrome in systemic juvenile rheumatoid arthritis successfully treated with cyclosporine. 7 Pediat 1996;128:275-8.

10 Fishman D, Rooney M, Woo P. Successful management of reactive haemophagocytic syndrome in systemic-onse juvenile chronic arthritis [letter]. Br F Rheumatol 1995;34: 888

11 Petty RE, Southwood TR. Classification of childhood arthritis: divide and conquer. F Rheumatol 1998;25:186970.

12 Petty RE, Southwood TR, Baum J, et al. Revision of the proposed classification criteria for juvenile idiopathic arthritis: Durban, 1997. F Rheumatol 1998;25:1991-4

13 Prieur AM, Roux-Lombard P, Dayer JM. Dynamics of fever and the cytokine network in systemic juvenile arthritis. Rev and the cytokine network in system

14 Henter JI, Arico M, Elinder G, et al. Familial hemophagocytic lymphohistiocytosis. Primary hemophagocytic lymphoh.

15 Writing Group of the Histiocytic Society. Histiocytosis syndromes in children. Lancet 1987;1:208.
16 Favara BE, Feller AC, Paulli M, et al. A contemporary classification of hist Paulli M, et al. A contemporary classification of histiocytic
Pediatr Oncol 1997;29:157.

17 Lawlor ER, Webb DW, Hill A, Wadsworth LD. Thrombotic thrombocytopenic purpura: a treatable cause of childhood encephalopathy. $\mathcal{F}$ Pediatr 1997;130:313-16.

18 Stephan JL, Zeller J, Hubert P, et al. Macrophage activation syndrome and rheumatic disease in childhood: a report of four new cases. Clin Exp Rheumatol 1993;11:451-6.

19 Koff RS, Makela AI, Koivikko A. Acute liver cell failure and encephalopathy (Reye's syndrome) during salicylate herapy. Acta Paediatr Scand 1975;64:877-81.

20 Silverman ED, Miller JJ, Bernstein B, Shafai T. Consumption coagulopathy associated with systemic juvenile rheumatoid arthritis. F Pediatr 1983;103:872-6.

21 Jacobs JC, Gorin LJ, Hanissian AS, et al. Consumption coagulopathy after gold therapy for JRA [letter]. $\mathcal{F}$ Pediatr 1984;105:674-5.

22 Schwartz D, Averbuch M, Pines A, et al. Disseminated intravascular coagulation with renal and liver damage as the predominant manifestations of recurrent relapses in systemic juvenile rheumatoid arthritis. Ann Rheum Dis 1992;3:347-9.

23 Henter JI, Elinder G, Soder O, et al. Hypercytokinemia in amilial hemophagocytic lymphohistiocytosis. Blood 1991; 78:2918-22

24 Akashi K, Hayashi S, Gondo H, et al. Involvement of interferon-gamma and macrophage colony stimulating factor in pathogenesis of haemophagocytic lymphohistiocytosis in adults. Br f Haematol 1994;87:243-50.

25 Schooley JC, Kullgren B, Allison AC Inhibition by interleukin-1 of the action of erythropoietin on erythroid precursors and its possible role in the pathogenesis of precursors and its possible role in the pathogenesis
hypoplastic anaemias. Br F Haematol 1987;67:11-17.

26 Reinherz EL, O'Brien C, Rosenthal P, Schlossman SF. The cellular basis for viral-induced immunodeficiency: analysis by monoclonal antibodies. F Immunol 1980;125:1269-74.

27 Scott JP, Gerber P, Maryjowski MC, Pachman LM. Evidence for intravascular coagulation in systemic onset, but not polyarticular, juvenile rheumatoid arthritis. Arthritis Rheum 1985;28:256-61.

28 Mukamel M, Bernstein BH, Brik R, Lehman TJ. The prevalence of coagulation abnormalities in juvenile rheumatoid arthritis. F Rheumatol 1987;14:1147-9.

29 Gogusev J, Nezelof C. Malignant histiocytosis. Histologic, cytochemical, chromosomal, and molecular data with a nosologic discussion. Hematol Oncol North Am 1998;12: 445-63.

30 Murphy SB. Cyclosporine in activated macrophage and histiocytic syndromes [letter; comment]. F Pediatr 1997;130 1012 .

31 De Benedetti F, Pignatti P, Massa M, et al. Soluble tumor necrosis factor receptor levels reflect coagulation abnormalities in systemic juvenile chronic arthritis. Brf Rheumatol 1997;36:581-8.

32 Pralahad S, Lovell DJ, Grom AA. Use of etanercept in the treatment of macrophage activation syndrome. Arthritis Rheum 2000;9(suppl):S257.

33 Albert A, Azgui Z, Buisine J, et al. Macrophage activation syndromes. Nouv Rev Fr Hematol 1992;34:435-41.

34 Stepp SE, Mathew PA, Bennett M, et al. Perforin: more than just an effector molecule. Immunol Today 2000;21:254-6.

35 Normand NJ, Lehman TJA, Elkon KB, Onel KB. Lower expression of perforin in CD8+ T cells of patients with systemic onset juvenile rheumatoid arthritis. Arthritis Rheum 2000;9(suppl):S406. 